\title{
Cochrane Review Summary: Community-based intervention packages for reducing maternal and neonatal morbidity and mortality and improving neonatal outcomes
}

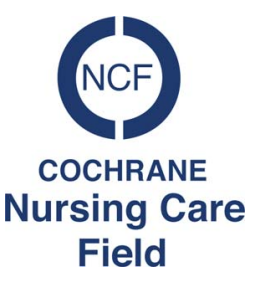

\section{Daksha Trivedi}

Senior Research Fellow, Evidence Based Practice, Centre for Research in Primary and Community Care, University of Hertfordshire, Hatfield, UK

Keywords: community; interventions; morbidity; mortality; maternal; neonatal; perinatal deaths; stillbirths

First published online 18 April 2016

\section{Review question}

Are community-based intervention packages effective in reducing maternal and neonatal morbidity and mortality, and improving neonatal outcomes?

\section{Relevance to primary care and nursing}

Primary health-care professionals, particularly nurses, have a key role in improving maternal health and reducing child mortality (Department of Health, 2010; Department of Health, Partnerships for Children, Families and Maternity, 2011).

\section{Characteristics of the evidence}

This Cochrane review contained 26 randomisedcontrolled trials (RCTs), of which 24 were cluster randomised, one was a controlled clinical cluster trial and one a quasi-RCT, targeting women of reproductive age, mainly pregnant women at any period of gestation (Lassi and Bhutta, 2015). They were mostly conducted in developing countries with one study based in Greece and covered a wide range of intervention packages delivered in

Correspondence to: Dr Daksha Trivedi, Senior Research Fellow, Evidence Based Practice, Centre for Research in Primary and Community Care, University of Hertfordshire, College Lane, Hatfield, AL10 9AB, UK. Email: d.trivedi@ herts.ac.uk

(C) Cambridge University Press 2016 community settings. These included training of health-care workers [such as community midwives, health visitors and workers ( $\mathrm{CHW}$ ) facilitators, traditional birth attendants (TBA) and outreach workers (community residents)] in maternal and neonatal care. Control groups received usual maternal and neonatal services.

\section{Summary of key evidence}

Approximately half the studies were overall at low risk of bias, with almost all studies having minimal selection bias due to cluster randomisation. Many studies however were rated as having unclear risk of bias regarding attrition and blinding and varied in follow-up periods. Meta-analysis was conducted where appropriate, although results were heterogeneous. Evidence is summarised according to outcomes. Average risk ratios (RR) are given with $95 \%$ confidence intervals (CI) for primary outcomes. The number of studies and participants are shown in parentheses.

\section{Primary outcome}

Maternal mortality (maternal deaths (death while pregnant or within 42 days of termination) per live birth)

There was a small reduction (11 studies, $n=167311$; RR $0.80,95 \%$ CI $0.64-1.00)$ but no 
significant impact for intervention packages that included support groups, packages that mobilised the community and conducted home visits or training packages for TBAs, followed by home visits.

\section{Neonatal mortality (neonatal deaths from any cause among total live births)}

There was a significant reduction in overall mortality by $25 \%$ (21 studies, $n=302464$; RR $0.75,95 \%$ CI $0.67-0.83$ ) and significant reductions in early (deaths in first week of life) (11 studies, $n=131017$; RR 0.67; 95\% CI 0.58-0.77) and late (deaths from seven to 28 days of life) (11 studies, $n=148$ 822; RR 0.74; 95\% CI 0.65-0.86) neonatal mortality. Community packages that included support/advocacy groups $(16 \%$, nine studies, $n=155509$; RR 0.84 , 95\% CI 0.73-0.96) were effective in reducing total and early deaths. Community mobilisation (CM) with added homebased neonatal care significantly reduced total neonatal deaths (one study, $n=4248$; RR 0.66, 95\% CI 0.47-0.93).

\section{Secondary outcomes}

Perinatal mortality, stillbirths, maternal morbidity, iron/folate supplementation and tetanus immunisation, referral to health facility, skilled birth attendance, use of clean delivery kits, birthweight and breastfeeding rates, infant's weight and height.

$\mathrm{CM}$, education and home visits by $\mathrm{CHWs}$, which focussed on antenatal/early newborn care can reduce perinatal deaths (by 22\%) and stillbirths (by 19\%). Packages that consisted of CM and involved midwives visits significantly reduced stillbirths (by $46 \%$ ). Whilst packaged interventions can improve breastfeeding and health-care seeking for neonatal morbidities, the studies were very heterogeneous. However, antepartum newborn care and breastfeeding education to mothers doubled the rates of initiation of breastfeeding. The interventions can be effective on tetanus immunisation, use of clean delivery kits, improving rates for institutional deliveries but no overall impact on increasing skilled birth attendance.

\section{Implications for practice}

Community-based interventions can be packaged within existing maternal and newborn care and delivered through a range of community health workers. Family involvement through community support and advocacy groups and CM and education strategies, home visits by trained $\mathrm{CHWs}$ and improving referrals are particularly important.

\section{Implications for research}

More high-quality studies are required to examine costs of delivering community-based packages, as well as evaluate effectiveness in other settings.

\section{Acknowledgements}

The author is a member of the Cochrane Nursing Care Field (CNCF).

\section{Financial Support}

This research received no specific grant from any funding agency, commercial or not-for-profit sectors.

\section{Conflicts of Interest}

None.

\section{References}

Department of Health. 2010: Tackling health inequalities in infant and maternal health outcomes. Report of the Infant Mortality National Support Team. Retrieved 26 January 2016 from https://www.gov.uk/government/uploads/system/ uploads/attachment_data/file/215869/dh_122844.pdf.

Department of Health, Partnerships for Children, Families and Maternity. 2011: NHS at home: Children's Community Nursing Services. Retrieved 26 January 2016 from https://www. gov.uk/government/uploads/system/uploads/attachment_ data/file/215708/dh_124900.pdf.

Lassi, Z.S. and Bhutta, Z.A. 2015: Community-based intervention packages for reducing maternal and neonatal morbidity and mortality and improving neonatal outcomes. Cochrane Database of Systematic Reviews Issue 3, Art. No. CD007754. doi: 10.1002/14651858.CD007754.pub3. 